\title{
Predicting the outcome of shunt surgery in normal pressure hydrocephalus
}

\author{
Kaya Kilic
}

Received: 2 October 2008/Accepted: 2 December 2008 / Published online: 8 January 2009

(C) Springer-Verlag 2008

\section{Dear Sir,}

I read with great interest the article entitled "Continuous intraventricular pressure monitoring for diagnosis of normal-pressure hydrocephalus" by W.K. Pfisterer in the October 2007 issue of the journal (149(10): 983-90). We, neurosurgeons are all deeply concerned by the accurate diagnosis and treatment of this interesting syndrome from its first definition in the mid sixties. This article contributes in many ways to our traditional knowledge on the outcomes of the treatment of normal pressure hydrocephalus: long-term results are always welcome and the low morbidity rate of such an invasive investigation as intraventricular pressure monitoring is reassuring. Nevertheless, I disagree with the statement that "diagnosis of Normal-pressure hydrocephalus (NPH) by clinical and radiological findings alone is unreliable". Our long term results on the subject (1) which seem encouraging, is the reason of my objection. We studied the effectiveness of the repeated lumbar CSF tap test (RTT), lumbar external CSF drainage (LED) and radioisotope cisternography (RIC) in predicting the outcome of shunt surgery, as well as the diagnostic and prognostic value of the classic clinical triad in normal pressure hydrocephalus. Patients, 270, were referred to the Departments of Neurosurgery, in Nancy, France and in Istanbul, Turkey. Following all shunt procedures, postoperative assessments verified improvements in $88 \%$ of the RTT group and $91 \%$ of the LED group. Gait disturbance had improved in $90 \%$ at the end of

K. Kilic $(\bowtie)$

Haydarpasa Numune Education and Research Hospital,

Uskudar,

Istanbul, Turkey

e-mail: kayakilic@yahoo.com the second and 12th month follow-up. Cognitive dysfunction had improved in $79 \%$ at the second and in $77 \%$ at the 12th month follow-up. Urinary incontinence had improved in $66 \%$ at the second and in $62 \%$ at the 12th month follow-up.

From the surgical point of view, in this pathology, the greatest difficulty is not to make the diagnosis, but rather to identify the appropriate patients to operate on.

I also want to attract the attention to the fact that lumbar infusion tests and continuous ICP recording can all show that CSF dynamics is 'deficient or malfunctioning'; they may even be able to establish that the diagnosis is NPH, but none can confirm directly whether the patient will benefit from surgery. In other words, RTT and LED, instead of answering the question, that is of secondary importance to both surgeon and patient, 'does the patient have NPH or not', rather responds directly to the question of primary importance: 'will the patient benefit from this operation, and should he/she consequently, undergo surgery'. Therefore, the prediction of post-operative-like results, as if surgery has been performed, becomes an invaluable foresight, an opportunity which cannot be encountered in any other situation within the field of neurosurgery.

I congratulate the authors for their invaluable contribution to the field.

Kaya KILIC, M.D., Ph.D.

Department of Neurosurgery,

Haydarpasa Numune Education and Research Hospital, Uskudar, Istanbul, Turkey

1) Kilic K., Czorny A., Auque J., Berkman Z. Predicting the outcome of shunt surgery in normal pressure hydrocephalus. Journal of Clinical Neuroscience 2007; 14:729-736 